\title{
Prevalence of metallo- $\beta$-lactamase-encoding genes among carbapenem-resistant Pseudomonas aeruginosa strains isolated from burn patients in Iran
}

\author{
Fereshteh Jabalameli ${ }^{[1]}$, Elahe Taki ${ }^{[1]}$, Mohammad Emaneini ${ }^{[1]}$ and Reza Beigverdi ${ }^{[1]}$
}

[1]. Department of Microbiology, School of Medicine, Tehran University of Medical Sciences, Tehran, Iran.

\begin{abstract}
Carbapenem-resistant Pseudomonas aeruginosa (CRPA) has been considered a major cause of infection and mortality in burn patients, especially in developing countries such as Iran. One of the most common mechanisms of carbapenem resistance is production of metallo- $\beta$-lactamases [(MBLs), including Verona Integron-encoded Metallo-beta-lactamase (VIM), imipenemase (IMP), São Paulo metalo-beta-lactamase (SPM), German imipenemase (GIM), New Delhi metallo-beta-lactamase (NDM), Dutch imipenemase (DIM), AIM, Seoul imipenemase (SIM), KHM, Serratia metallo- $\beta$-lactamase (SMB), Tripoli metallo- $\beta$-lactamase (TMB), and Florence imipenemase (FIM)]. Limited information is available on the prevalence of CRPA and MBLs in Iranian burn units. We performed a systematic search by using different electronic databases, including Medline (via PubMed), Embase, Web of Science, and Iranian Database. Of 586 articles published from January 2000 to December 2016, 14 studies reporting the incidence of CRPA and MBLs as detected by molecular methods in burn patients were included in this review. The meta-analyses showed that the prevalence of CRPA, IMP, and VIM was 76.8\% (95\% CI 67.5-84.1), 13.1\% (95\% CI 4.7-31.5), and 21.4\% (95\% CI 14.630.1), respectively, in Iranian burn centers and remaining MBLs types have not yet been detected. There was a high prevalence of MBLs and CRPA in Iranian burn centers. Therefore, these measurements should be applied nationally and rigorous infection control measures and antimicrobial stewardship will be the major pillars to control multidrug resistant microorganisms, such as CRPA.
\end{abstract}

Keywords: MBLs. CRPA. Burn. Iran.

\section{INTRODUCTION}

Pseudomonas aeruginosa is a major cause of life-threatening infections in burn patients worldwide ${ }^{1,2}$. It has a remarkable capacity to develop resistance to multiple classes of antimicrobial agents, and consequently, is considered a multidrug resistant (MDR) pathogen ${ }^{3}$. Carbapenems are considered first-line agents to treat severe cases of $P$. aeruginosa infections $s^{4}$. In spite of this, carbapenem-resistant $P$. aeruginosa (CRPA) has been increasing in recent years, which is associated with high mortality, morbidity, long hospital stays, and increased costs ${ }^{1,5,6}$. One of the most common mechanisms of carbapenem resistance is production of metallo- $\beta$-lactamases (MBLs) ${ }^{6}$. MBL enzymes are also able to hydrolyze penicillin and cephalosporins? ${ }^{7}$. Twelve different types of MBLs (VIM, IMP, SPM, GIM, NDM, DIM, AIM, SIM, KHM, SMB, TMB, and FIM) have been identified so $\mathrm{far}^{3}$. These genes are usually encoded by mobile genetic elements, such as plasmids, transposons, and integrons, that allow them to disseminate horizontally among Gram-negative bacteria, posing a global challenge for all countries ${ }^{3,6}$. MBL-producing

Corresponding author: $\mathrm{Dr}^{\mathrm{a}}$ Reza Beigverdi

e-mail: r-beigverdi@tums.ac.ir

Received 3 february 2018

Accepted 8 june 2018
P. aeruginosa was originally discovered in 1991 in Japan. Since then, it has been described in different parts of the world $3,5,6,8$. The first MBL-positive strains of $P$. aeruginosa were described in an Iranian burn center in 2008 and are currently recognized as one of the crucial hospital-acquired infectious agents ${ }^{5}$. In Iranian burn centers, the prevalence of CRPA increased from $41 \%$ in 2008 to $57.4 \%$ in $2016^{1,5}$. To date, there are several reports from two different Iranian burn centers on the prevalence of MBLs among $\mathrm{CRPA}^{5,9-12}$. However, most of these studies only reported local information, and no systematic study has yet been performed. The aim of this surveillance review was to assess the prevalence of MBLs among CRPA in Iranian burn centers using a systematic review and meta-analysis according to the Preferred Reporting Items for Systematic Reviews and Meta-Analyses statement.

\section{METHODS}

\section{Search strategies}

We carried out a literature search of Medline (via PubMed), Embase, Web of Science, and Iranian Database from January 2000 to December 2016 using the following terms: Pseudomonas aeruginosa or P. aeruginosa and Metallo- $\beta$-lactamases or MBLs and burn patients in combination with Iran. Cross-sectional or cohort studies that reported the prevalence of MBLs in burn patients were considered. The titles and abstracts for possible inclusion in 
the reviews were screened by two independent reviewers. Reviews were restricted to studies published in English and Persian languages and revealed the prevalence or incidence of CRPA and MBLs genes in burn patients. Eligible articles were selected based on three stages: title, abstracts, and full-text publication. Studies with the following characteristics were included: studies conducted only on burn patients and reporting the frequency of carbapenem resistance and MBLs genes, detection of MBLs genes [Verona Integron-encoded Metallobeta-lactamase (VIM), imipenemase (IMP), São Paulo metalobeta-lactamase (SPM), German imipenemase (GIM), New Delhi metallo-beta-lactamase (NDM), Dutch imipenemase (DIM), AIM, Seoul imipenemase (SIM), KHM, Serratia metallo$\beta$-lactamase (SMB), Tripoli metallo- $\beta$-lactamase (TMB), and Florence imipenemase (FIM)], by molecular methods [polymerase chain reaction (PCR)]. Studies that had one or more of the following characteristics were excluded: studies using phenotypic methods, duplicate and overlapping studies, studies published in languages other than English or Persian, studies with other than burn patients, studies that did not report CRPA and MBLs prevalence, nonhuman studies, review articles, congress abstracts, meta-analyses, or systematic reviews as well as articles available only in abstract form.

\section{Data extraction and definitions}

The following data were extracted from each study: name of first author, year of publication, year of study, study setting, number of cases investigated, study methods, sample size, and prevalence of CRPA and MBLs genes. Furthermore, to minimize the potential bias caused by an inadequate sample size, articles with less than 50 subjects were omitted. Two reviewers independently extracted all data from included studies, and a third reviewer reviewed results. Disagreements between the reviewers were resolved by consensus.

\section{Quality assessment of studies}

Two reviewers using a checklist provided by the Joanna Briggs Institute assessed the study quality independently ${ }^{13}$.

\section{Statistical analysis}

Meta-analysis was performed by Comprehensive MetaAnalysis (Biostat V2.2) software. We reported the amount of residual heterogeneity using the $\mathrm{I}^{2}$ statistic and Cochran's Q statistic to test the heterogeneity between inquiries. In order to assess any possible publication bias, Begg's rank correlation and Egger's weighted regression methods in combination with a funnel plot were used $(p<0.05$ was considered indicative of statistically significant publication bias).

\section{RESULTS}

Our literature search yielded 586 studies, and of these, 14 based on the mentioned criteria were included in the metaanalysis (Table 1). Figure 1 shows the study selection process and reasons for exclusion. Based on the 14 selected articles, the pooled prevalence of CRPA was $76.8 \%$ (95\% CI 67.5-84.1). The pooled prevalence of IMP and VIM among CRPA was $13.1 \%$ (95\% CI 4.7-31.5) and 21.4\% (95\% CI 14.6-30.1), respectively, as shown in Table 2. Heterogeneities between studies $\left(\mathrm{I}^{2}=95\right.$,

TABLE 1: Characteristics of studies included in the meta-analysis.

\begin{tabular}{|c|c|c|c|c|c|c|c|}
\hline Study $/^{*}$ & $\begin{array}{l}\text { Time of } \\
\text { stud }\end{array}$ & $\begin{array}{c}\text { Published } \\
\text { time }\end{array}$ & Province & $\begin{array}{c}\text { Sample } \\
\text { size }\end{array}$ & CRPA & \multicolumn{2}{|c|}{ Detection method (PCR) } \\
\hline Khosravi $^{5}$ & 2005-2006 & 2008 & Ahwaz & 100 & 41 & 0 & 8 \\
\hline Bahar $^{9}$ & $2007-2008$ & 2009 & Zanjan & 186 & 115 & 0 & 32 \\
\hline Saderi $^{12}$ & 2008 & 2010 & Tehran & 100 & 69 & 0 & 13 \\
\hline Sepehriseresht ${ }^{15}$ & 2008-2009 & 2012 & Tehran & 483 & 272 & 33 & 51 \\
\hline Mirsalehian ${ }^{10}$ & 2010 & 2010 & Tehran & 170 & 90 & 0 & 10 \\
\hline Neyestanaki ${ }^{11}$ & 2011-2012 & 2014 & Tehran & 214 & 100 & 3 & 55 \\
\hline Fallah $^{16}$ & 2012 & 2013 & Tehran & 83 & 83 & 6 & 0 \\
\hline Radan $^{19}$ & 2013-2014 & 2015 & Isfahan & 150 & 144 & 107 & NT \\
\hline Safari ${ }^{20}$ & 2014-2015 & 2016 & Isfahan & 150 & 144 & NT & 35 \\
\hline Sadredinamin ${ }^{21}$ & 2015-2016 & 2016 & Tehran & 100 & 95 & 13 & 0 \\
\hline Tarashi22 & 2016 & 2016 & Tehran & 309 & 278 & 30 & 52 \\
\hline
\end{tabular}

CRPA: carbapenem-resistant Pseudomona aeruginosa; PCR: polymerase chain reaction; IMP: imipenemase; VIM: Verona Integron-encoded metallo-beta-lactamase; NT: not detected. *Author and respectve reference. 


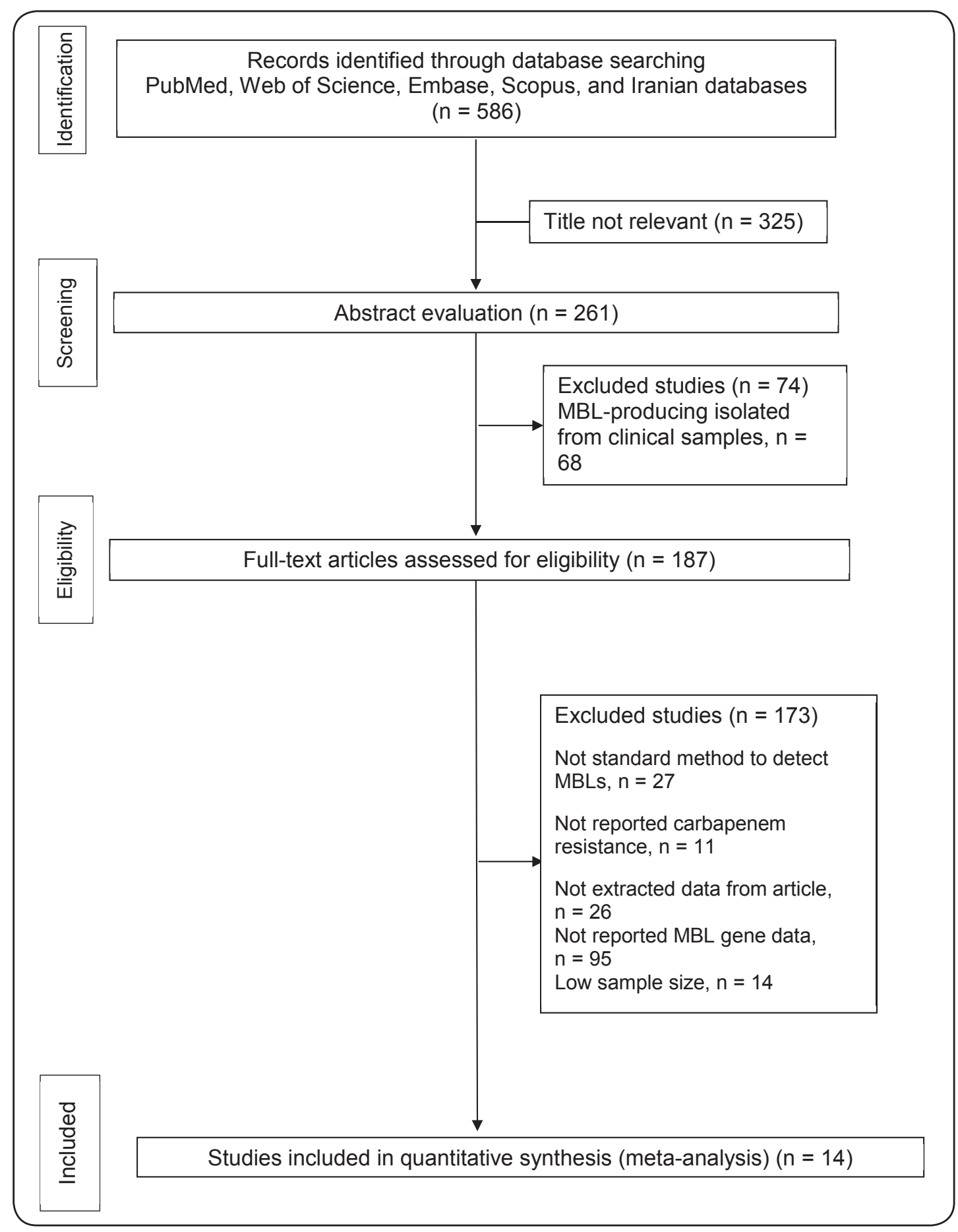

FIGURE 1: Summary of the literature search and study selection. MBLs: metallo- $\beta$-lactamases. 
TABLE 2: Meta-analysis of CRPA and MBLs prevalence among burn patients in Iran.

\begin{tabular}{|c|c|c|c|c|c|c|c|}
\hline Subgroup & Study (n) & Prevalence & $\mathrm{n} / \mathrm{N}$ & $\begin{array}{l}\text { Heterogeneity } \\
\text { test, I2 }\end{array}$ & $\begin{array}{l}\text { Heterogeneity } \\
\text { test, } \mathrm{P} \text {-value }\end{array}$ & $\begin{array}{l}\text { Begg's } \\
\text { test }\end{array}$ & Egger's test \\
\hline CRPA* & 14 & $76.8(67.5-84.1)$ & $1,701 / 2,450$ & 95 & $<.001$ & 0.02 & 0.004 \\
\hline IMP & 8 & $13.1(4.7-31.5)$ & $206 / 1,168$ & 97 & $<.001$ & 0.5 & 0.3 \\
\hline
\end{tabular}

CRPA: carbapenem-resistant Pseudomona aeruginosa; MBLs: metallo- $\beta$-lactamases; IMP: imipenemase; VIM: Verona Integron-encoded metallo-betalactamase; $\mathbf{n}$ : number of events (CRPA, IMP, and VIM); N: total number of Pseudomona aeruginosa isolated from burn patients.

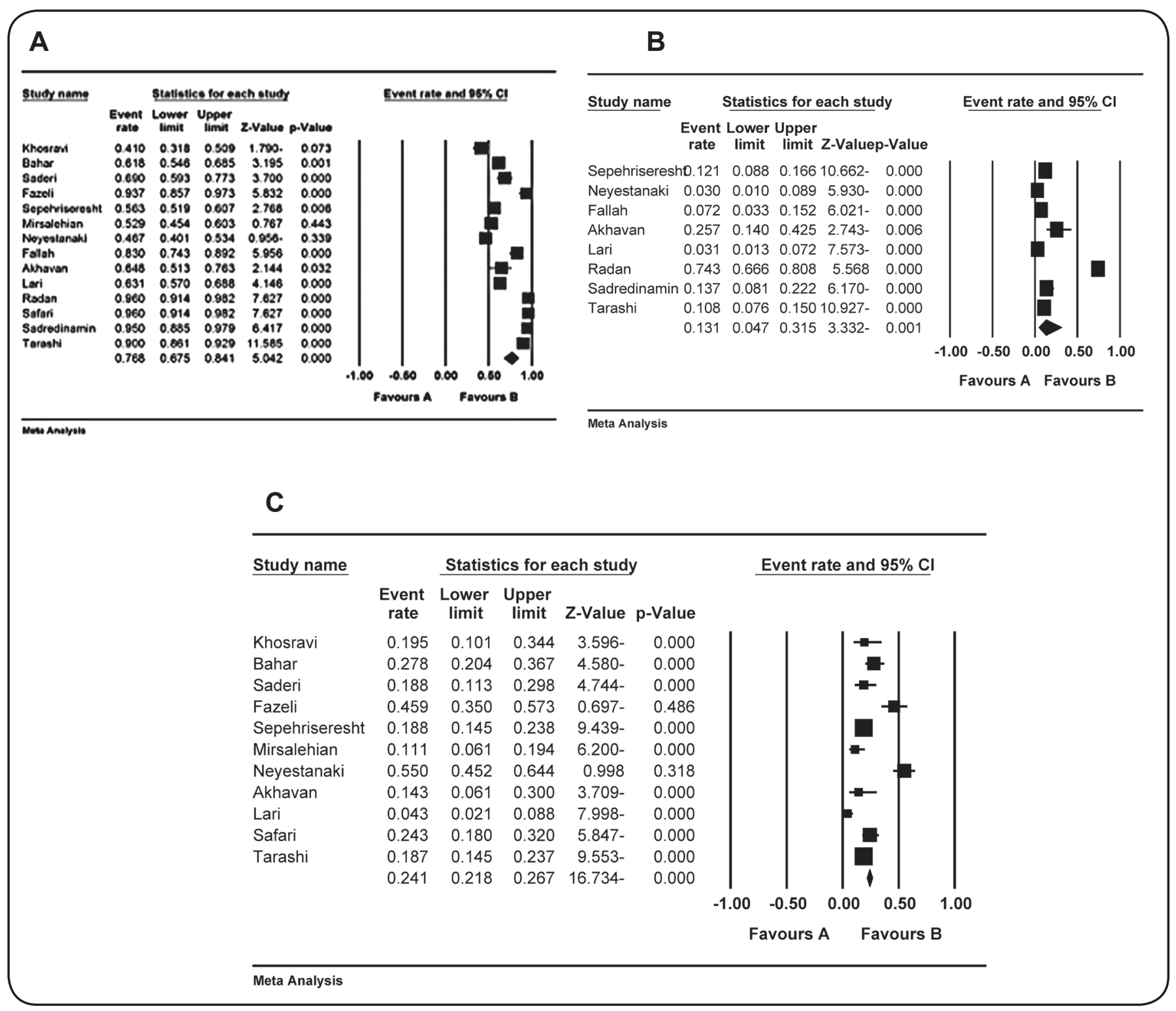

FIGURE 2: Forest plot of the studies included in this meta-analysis. (A) The prevalence of CRPA in burn patients. (B) and (C) The prevalence of IMP and VIM genes among CRPA, respectively. CRPA: carbapenem-resistant Pseudomona aeruginosa; IMP: imipenemase; VIM: Verona Integron-encoded metallobeta-lactamase. 
A

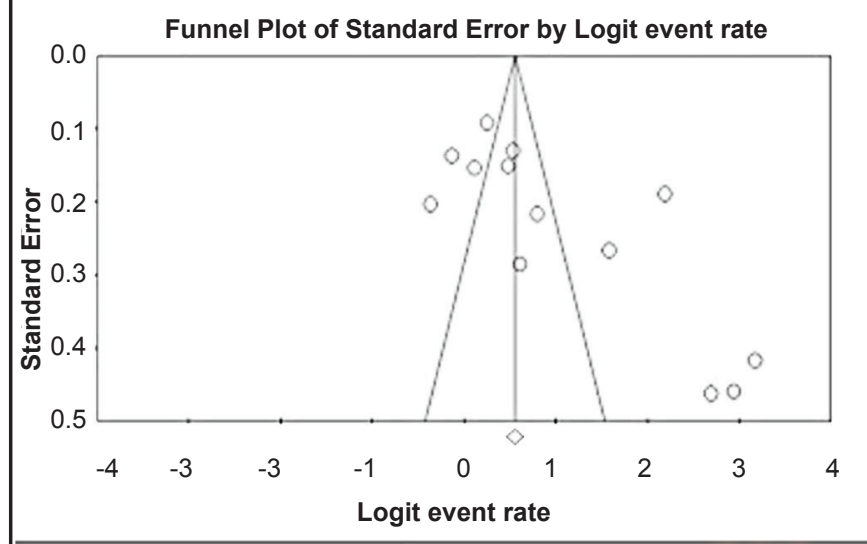

B

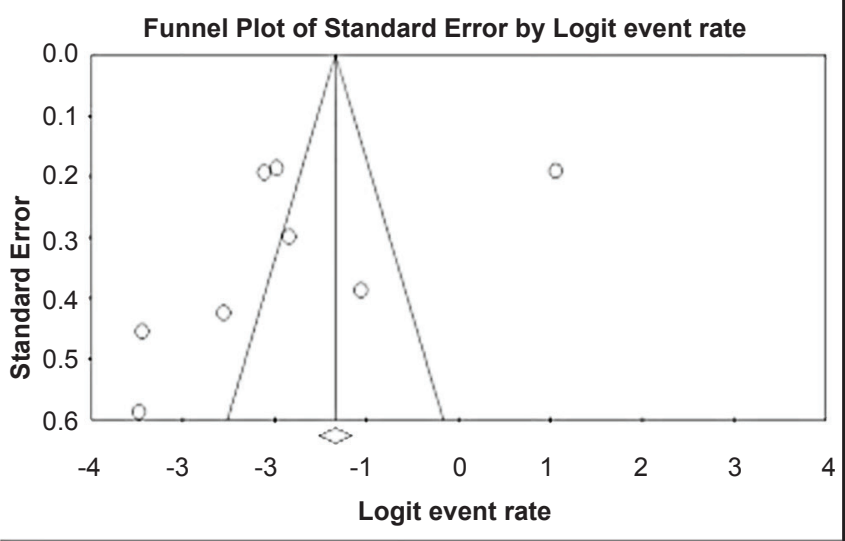

C

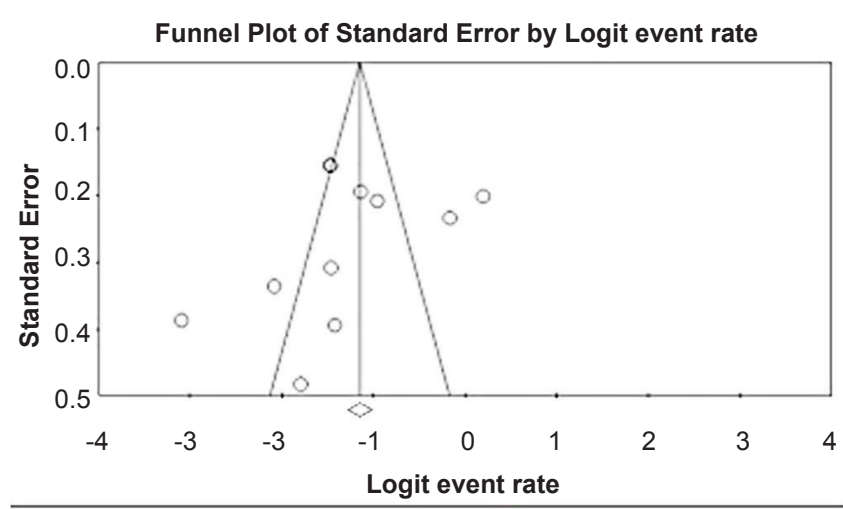

FIGURE 3: Funnel plot of publication bias for the included studies. (A) The prevalence of CRPA in burn patients. (B) and (C) The prevalence of IMP and VIM genes among CRPA, respectively. Asymmetric shape of funnel plots suggests bias in this meta-analysis. CRPA: carbapenem-resistant Pseudomona aeruginosa; IMP: imipenemase; VIM: Verona Integron-encoded metallo-beta-lactamase.

$\mathrm{p}<0.001$ for CRPA; $\mathrm{I}^{2}=97, \mathrm{p}<0.001$ for IMP; and $\mathrm{I}^{2}=91$, $\mathrm{p}<0.001$ for VIM) were found, so the random effect model was used for meta-analysis. Figure 2 shows forest plots for the prevalence rate of CRPA, IMP, and VIM. As it is shown in Table 2, some evidence of publication bias was observed by Begg's rank correlation test ( $\mathrm{p}=0.02$ for CRPA), but it was not observed for IMP and VIM genes $(p=0.5$ for IMP and $p=0.4$ for VIM). Some evidence of publication bias was detected by Egger's weighted regression ( $p=0.004$ for CRPA), but it was not detected for IMP and VIM genes $(p=0.3$ for IMP and $p=0.6$ for VIM). Asymmetric shapes of the funnel plots (Figure 3) show some evidence of publication bias among the evaluated papers.

\section{DISCUSSION}

To our knowledge, the current study was the first comprehensive systematic review addressing the prevalence of CRPA and MBLs genes in burn units in Iran. Based on the meta-analysis results, the pooled prevalence of CRPA, IMP, and
VIM genes in burn patients was 76.8\% (95\% CI 67.5-84.1), 13.1\% (95\% CI 4.7-31.5), and 21.4\% (95\% CI 14.6-30.1), respectively,9-12,14-22. Our analyses showed that the IMP and VIM genes were more prevalent in Iranian burn centers and remaining MBLs types have not yet been found. Our findings are similar to previous reports by other authors ${ }^{23-25}$. According to the analysis, it seems that the prevalence of CRPA and MBLs genes in Iranian burn units is higher than in industrialized countries, such as Norway and Sweden ${ }^{26}$. Several factors may explain this situation. First, most countries in Europe have an effective program of prevention and control of nosocomial infections ${ }^{27}$. While in developing countries, such as Iran, the hospital infection control committee may exist on paper, in practice, they barely exist ${ }^{28}$. Unfortunately, in Iran, infection control teams are inexperienced, inadequate, not well trained, and a clinical microbiologist is not included ${ }^{29,30}$. Hand hygiene is one of the simplest, most effective, and highly recommended infection control measures, but compliance with it is quite 
poor among Iranian healthcare workers ${ }^{30,31}$. Heavy workload (patient overcrowding), limited infrastructures, inadequate healthcare worker to patient ratios, behavioral aspects, skin irritation by hand hygiene products, and lack of salary are the major reasons for noncompliance ${ }^{30}$. Second, wards with a high bed occupancy rate are a major cause of poor attention to infection control protocols in Iranian burn units ${ }^{29,30}$. Third, lack of isolation rooms is another common problem in Iranian burn hospitals; thereby, patients infected with MBL-producing $P$. aeruginosa mingle with other patients in multi-bed rooms ${ }^{30}$. Fourth, the use of personal protective equipment (PPEs) such as gowns, gloves, and mask is a simple method for infection controls $^{29}$. In low-income countries, such as Iran, the use of PPEs is restricted owing to inadequate resources. In contrast, in the Netherlands, all hospitals are equipped with appropriate PPEs ${ }^{29}$. Fifth, in developing countries, such as Iran, inappropriate use of antibiotic and empiric therapies because of the lack of a good microbiological laboratory capacity is prevalent. Consequently, the emergence of resistant bacteria, such as CRPA, is accelerating in Iranian burn centers ${ }^{30}$. Unfortunately, in Iranian burn hospitals, routine detection of MBL production in P. aeruginosa is not performed. Therefore, we suggest that it is essential for carbapenem-resistant isolates to be screened for MBLs. Finally, lack of systems to monitor antibiotics prescribed by physicians and lack of a national stewardship have remained as a major challenge in Iranian burn units ${ }^{32}$. The present review had some limitations. First, the studies could not fully indicate the prevalence of MBLs among burn patients in Iran because the magnitude of MBLs infections was not yet determined in different areas of the country. Second, only published articles were considered in the present meta-analysis; hence, as in any other meta-analysis, the potential for publication bias should be considered. Third, heterogeneity was observed among the included studies. In conclusion, the prevalence of MBLs and CRPA was high in Iranian burn centers. Thereby, reducing antibiotic overuse, adherence to hand hygiene, early detection of MBLs producer isolates, education and training in antibiotic prescribing, environmental cleaning, contact precautions, and active surveillance are recommended strategies for prevention and spread of these strains.

\section{Acknowledgments}

This research has been supported by Tehran University of Medical Sciences and Health Services. Study Grant no: 32465/95-02-30.

\section{Conflict of interest}

The authors declare that there is no conflicts of interest.

\section{REFERENCES}

1. Khosravi AD, Shafie F, Abbasi Montazeri E, Rostami S. The frequency of genes encoding exotoxin A and exoenzyme $\mathrm{S}$ in Pseudomonas aeruginosa strains isolated from burn patients. Burns. 2016;42(5):1116-20.

2. Leseva M, Arguirova M, Nashev D, Zamfirova E, and Hadzhyiski O. Nosocomial infections in burn patients: etiology, antimicrobial resistance, means to control. Ann Burns Fire Disasters. 2013;26(1): $5-11$.

3. El-Domany RA, Emara M, El-Magd MA, Moustafa WH, Abdeltwab NM. Emergence of imipenem-resistant Pseudomonas aeruginosa clinical isolates from Egypt Coharboring VIM and IMP carbapenemases. Microb Drug Resist. 2017;23(6):682-6.

4. Dantas RC, Ferreira ML, Gontijo-Filho PP, Ribas RM. Pseudomonas aeruginosa bacteraemia: independent risk factors for mortality and impact of resistance on outcome. J Med Microbiol. 2014;63 (Pt 12:1679-87.

5. Khosravi AD, and Mihani F. Detection of metallo-beta-lactamaseproducing Pseudomonas aeruginosa strains isolated from burn patients in Ahwaz, Iran. Diagn Microbiol Infect Dis. 2008;60(1): $125-8$.

6. Malkocoglu G, Aktas E, Bayraktar B, Otlu B, Bulut ME. VIM1, VIM-2, and GES-5 Carbapenemases Among Pseudomonas aeruginosa Isolates at a Tertiary Hospital in Istanbul, Turkey. Microb Drug Resist. 2017;23(3):328-34.

7. Jeon JH, Lee JH, Lee JJ, Park KS, Karim AM, Lee CR et al. Structural basis for carbapenem-hydrolyzing mechanisms of carbapenemases conferring antibiotic resistance. Int $\mathrm{J}$ Mol Sci. 2015;16(5):9654-92.

8. Yousefi S, Farajnia S, Nahaei MR, Akhi MT, Ghotaslou R, Soroush $\mathrm{MH}$ et al. Detection of metallo-beta-lactamase-encoding genes among clinical isolates of Pseudomonas aeruginosa in northwest of Iran. Diagn Microbiol Infect Dis. 2010;68(3):322-5.

9. Bahar MA, Jamali S, Samadikuchaksaraei A. Imipenem-resistant Pseudomonas aeruginosa strains carry metallo-beta-lactamase gene bla(VIM) in a level I Iranian Burn Hospital. Burns. 2010;36(6):826-30.

10. Mirsalehian A, Nakhjavani F, Bahador A, Jabal ameli F, Bigverdi R, Goli H. 2011. Prevalence of MBL-producing Pseudomonas aeruginosa isolated from burn patients. TUMS (Persian). 2011;68(10):563-9.

11. Neyestanaki DK, Mirsalehian A, Rezagholizadeh F, Jabalameli F, Taherikalani M, Emaneini M. Determination of extended spectrum beta-lactamases, metallo-beta-lactamases and AmpC-betalactamases among carbapenem resistant Pseudomonas aeruginosa isolated from burn patients. Burns. 2014;40(8):1556-61.

12. Saderi H, Lotfalipour H, Owlia P, Salimi H. Detection of metallo- $\beta$ lactamase producing Pseudomonas aeruginosa isolated from burn patients in Tehran, Iran. Laboratory Medicine. 2010;41(10):609-12.

13. Moher D, Liberati A, Tetzlaff J, Altman DG, PRISMA Group. Preferred reporting items for systematic reviews and meta-analyses: the PRISMA statement. PLoS Med. 2009;6(7):e1000097.

14. Fazeli H, Moslehi Z, Irajian G, Salehi M. Determination of drug resistance patterns and detection of bla-VIM gene in Pseudomonas aeruginosa strains Isolated from burned patients in the Emam Mosa Kazem hospital, Esfahan, Iran (2008-9). IJMM (Persian). 2010;3(4):1-8.

15. Sepehriseresht S, Boroumand MA, Pourgholi L, Sotoudeh Anvari M, Habibi E, Sattarzadeh Tabrizi M. Detection of vim- and ipmtype metallo-beta-lactamases in Pseudomonas aeruginosa clinical isolates. Arch Iran Med. 2012;15(11):670-3.

16. Fallah F, Borhan RS, Hashemi A. Detection of bla (IMP) and bla (VIM) metallo- $\beta$-lactamases genes among Pseudomonas aeruginosa strains. Int J Burns Trauma. 2013;3(2):122.

17. Akhavan-Tafti F, Eslami G, Zandi H, Mousavi SM, Zarei M. Prevalence of blaVIM, blaIPM and blaNDM metallo-betalactamases enzymes in Pseudomonas aeruginosa isolated from burn wounds in Shahid Sadoughi Burn Hospital in Yazd. J Isfahan Med Sch. 2014;31(263):1955-64. 
18. Lari AR, Azimi L, Soroush S, Taherikalani M. Low prevalence of metallo-beta-lactamase in Pseudomonas aeruginosa isolated from a tertiary burn care center in Tehran. Int J Immunopathol Pharmacol. 2015;28(3):384-9.

19. Radan M, Moniri R, Khorshidi A, Gilasi H, Norouzi Z, Beigi F, et al. 2016. Emerging carbapenem-resistant Pseudomonas aeruginosa isolates carrying blaIMP among burn patients in Isfahan, Iran. Arch Trauma Res. 2016;5(3): e33664.

20. Saffari M, Firoozeh F, Pourbabaee M, Zibaei M. Evaluation of metallo-beta-lactamase-production and carriage of bla-VIM Genes in Pseudomonas aeruginosa isolated from burn wound Infections in Isfahan. Arch Trauma Res. 2016;5(4):e34343.

21. Sadredinamin M, Hashemi A, Goudarzi H, Tarashi S, Yousefi Nojookambari N, Taki E. Detection of blaIMP, blaVIM and OprD genes among Pseudomonas aeruginosa isolated from burn patients. JMUMS (Persian). 2016;26(138):181-6.

22. Tarashi S, Goudarzi H, Erfanimanesh S, Pormohammad A, Hashemi A. Phenotypic and molecular detection of metallo-beta-lactamase genes among imipenem resistant Pseudomonas aeruginosa and Acinetobacter baumannii strains isolated from patients with burn injuries. Arch Clin Infect Dis. 2016;11(4):e39036

23. Kouda S, Ohara M, Onodera M, Fujiue Y, Sasaki M, Kohara T, et al. Increased prevalence and clonal dissemination of multidrugresistant Pseudomonas aeruginosa with the blaIMP-1 gene cassette in Hiroshima. J Antimicrob Chemother. 2009;64(1):46-51.

24. Mansour W, Poirel L, Bettaieb D, Bouallegue O, Boujaafar N, Nordmann P. Metallo-beta-lactamase-producing Pseudomonas aeruginosa isolates in Tunisia. Diagn Microbiol Infect Dis. 2009;64(4):458-61.

25. Meradji S, Barguigua A, Bentakouk MC, Nayme K, Zerouali K, Mazouz D, et al. Epidemiology and virulence of VIM-4 metallo- beta-lactamase-producing Pseudomonas aeruginosa isolated from burn patients in eastern Algeria. Burns. 2016;42(4):906-18.

26. Samuelsen O, Toleman MA, Sundsfjord A, Rydberg J, Leegaard TM, Walder M, et al. Molecular epidemiology of metallo-betalactamase-producing Pseudomonas aeruginosa isolates from Norway and Sweden shows import of international clones and local clonal expansion. Antimicrob Agents Chemother. 2010;54(1):34652.

27. Zhang S, Sun X, Chang W, Dai Y, Ma X. Systematic review and meta-analysis of the epidemiology of vancomycin-intermediate and heterogeneous vancomycin-intermediate Staphylococcus aureus isolates. PLoS One. 2015;10(8):e0136082.

28. Ider BE, Adams J, Morton A, Whitby M, Clements A. Perceptions of healthcare professionals regarding the main challenges and barriers to effective hospital infection control in Mongolia: a qualitative study. BMC Infect Dis. 2012;12:170.

29. Alp E, Damani N. Healthcare-associated infections in intensive care units: epidemiology and infection control in low-to-middle income countries. J Infect Dev Ctries. 2015;9(10):1040-5.

30. Emaneini M, Hosseinkhani F, Jabalameli F, Nasiri MJ, Dadashi M, Pouriran R, et al. Prevalence of vancomycin-resistant Enterococcus in Iran: a systematic review and meta-analysis. Eur J Clin Microbiol Infect Dis. 2016;35(9):1387-92.

31. Alp E, Leblebicioglu H, Doganay M, Voss A. Infection control practice in countries with limited resources. Ann Clin Microbiol Antimicrob. 2011;10:36.

32. Esfandiari A, Rashidian A, Masoumi Asl H, Rahimi Foroushani A, Salari H, Akbari Sari A. Prevention and control of health care-associated infections in Iran: A qualitative study to explore challenges and barriers. Am J Infect Control. 2016;44(10):1149-53. 